\title{
Volumic Power Variation with Temperature in a Pure Iron Billet Placed in an Induction Furnace
}

\author{
MEROUANE Boidjemaa ${ }^{1, a}$, SEBBANI Mohamed $^{2, b}$
}

${ }^{1}$ Electroheat Laboratory, University of Sciences and Technology of Oran, 31000 Oran, Algeria

${ }^{2}$ University of Essenia-Oran, Department of Physics, 31000 Oran , Algeria

aemail: merouaneboudjemaa@yahoo.fr

bemail: msebbani@yahoo.fr

Keywords: iron billet, the induction heating, magnetic materials

Abstract: We study a pure iron billet placed in an induction furnace. The magnetic reluctivity of pure iron varies strongly according to the temperature. The volumic power and total current in the billet increase linearly with temperature. But, before and near the Curie point, those parameters increase very strongly. This, has his importance in the conception of the induction heating of magnetic materials.

\section{Maxwell equations and modelisation of the magnetization of saturation with temperature}

For a homogeneous and isotropic material (pure iron), the permittivity $\varepsilon$, the permeability $\mathrm{m}$, the conductivity $\gamma$ are expressed by constants only if the material is linear. In particular, the permeability $\mathrm{m}$ is a non-linear function of the applied field $\mathrm{H}_{0}$ without matter and temperature $\mathrm{T}$. Then, the Maxwell's equations are:

$$
\begin{aligned}
& \operatorname{rot} \vec{E}=-\frac{\partial \vec{B}}{\partial t} \\
& \operatorname{rot} \vec{H}=\vec{J}+\frac{\partial \vec{D}}{\partial t} \\
& \operatorname{div} \vec{B}=0 \\
& \operatorname{div} \vec{D}=\rho \\
& \vec{D}=\varepsilon \vec{E} \\
& \vec{J}=\gamma \vec{E} \\
& \vec{B}=\mu\left(H_{0}, T\right) \cdot \vec{H}=\mu_{0} \mu_{r}\left(H_{0}, T\right) \cdot \vec{H}=\mu_{0}\left[\vec{H}_{0}+\vec{M}\left(H_{0}, T\right)\right] \\
& \vec{M}\left(H_{0}, T\right)=\chi\left(H_{0}, T\right) \cdot \vec{H}_{0}
\end{aligned}
$$

Combining (7) and (8), one obtains:

$\mu_{r}\left(H_{0}, T\right)=1+\chi\left(H_{0}, T\right)=\frac{1}{v_{r}\left(H_{0}, T\right)}$

where $\vec{E}=$ electric field, $\vec{J}=$ total current density, $\gamma=$ electric conductivity, $\vec{D}=$ electric induction, $\rho=$ volumic and superficial density of charge, $\varepsilon=$ absolute permittivity, $\vec{B}=$ magnetic induction, $\vec{H}=$ mag-netic field, $H_{0}=$ applied magnetic field, $\vec{M}=$ magne-tization density, $\mu$ and $\mu_{r}=$ absolute and relative permeabilities, $v_{r}=$ relative reluctivity, $\chi=$ magne-tic susceptibility.

Using the Brillouin's function, [1], [3], the theory of ferromagnetism and law of Curie-Weiss below the temperature of Curie, the variation of the magnetization $M$ according to the applied field $H_{0}$ and the temperature $\mathrm{T}$ was obtained [2]. The magnetization of saturation $M_{S}$ corresponds to $H_{0}=0$ and $\mathrm{T}=0$. The reduced magnetization of saturation is defined as:

$$
m_{s}=\frac{M_{S}}{N \cdot \mu_{B}}
$$


where $\mathrm{N}=$ number of atoms of pure iron per unit volume, $\mu_{B}=$ Bohr's magneton. The equation of $m_{s}$ is [2]) :

$m_{S}=\frac{2 J+1}{2} g \cdot \operatorname{coth}\left(\frac{2 J+1}{2} \cdot \frac{3 T_{C}}{(J+1) g} \cdot \frac{m_{S}}{T}\right)-\frac{g}{2} \cdot \operatorname{coth}\left(\frac{1}{2 J} \cdot \frac{3 T_{C}}{(J+1) g} \cdot \frac{m_{S}}{T}\right)$

where $\mathrm{J}=$ magnetic quantum number, $\mathrm{g}=2.0023$,fac-tor of spectral decomposition, $T_{C}=1043 \mathrm{~K}$ =tempera-ture of Curie for the pure iron. The equation (11) is a transcendant equation in $m_{s}$. With all magnetothermal data concerning pure iron, [2] , this equation takes the form:

$m_{S}=3.221150 \cdot \operatorname{coth}\left(1075.262377 \cdot \frac{m_{S}}{T}\right)-1.00150 \cdot \operatorname{coth}\left(334.197081 \cdot \frac{m_{S}}{T}\right)$

It was solved for $T \in\left[0, T_{C}\right]$ using the software Maple. Figure 1 represents $m_{s}=f(T)$.

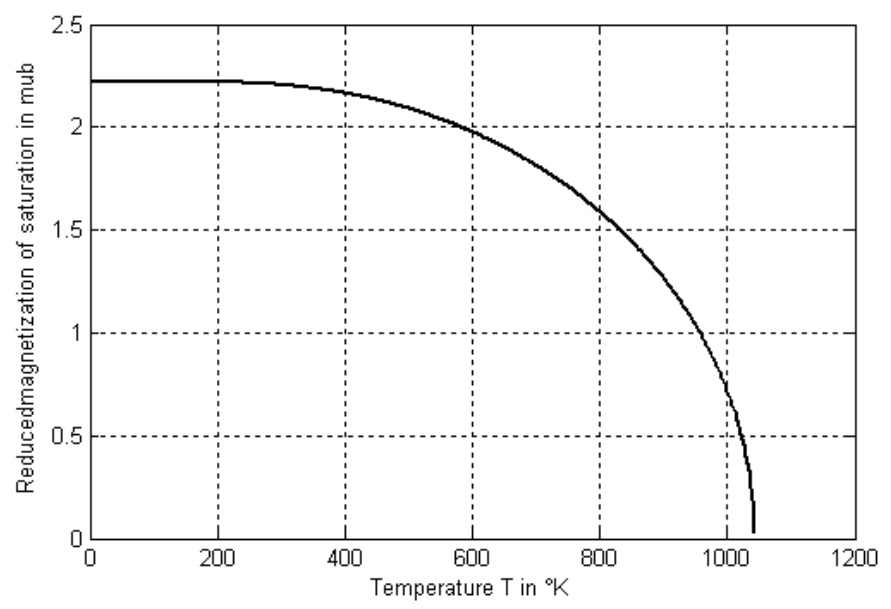

Figure 1. Reduced magnetization of saturation in $\mathrm{m}_{\mathrm{B}}$ versus temperature

\section{Studied system and equations}

\subsection{Studied system}

This system includes the inductor and the load. The inductor, in copper, has a diameter $\mathrm{d}_{\mathrm{i}}=6 \mathrm{~mm}$ and a height $h_{i}=19.25 \mathrm{~mm}$. The current in it is $6600 \mathrm{~A}, 60 \mathrm{~Hz}$. The load is a pure iron cylinder (billet). Its diameter is $d_{b}=25 \mathrm{~mm}$ and its height is $h_{b}=84.25 \mathrm{~mm}$. Between the inductor and load exists a gap of $12.5 \mathrm{~mm}$.

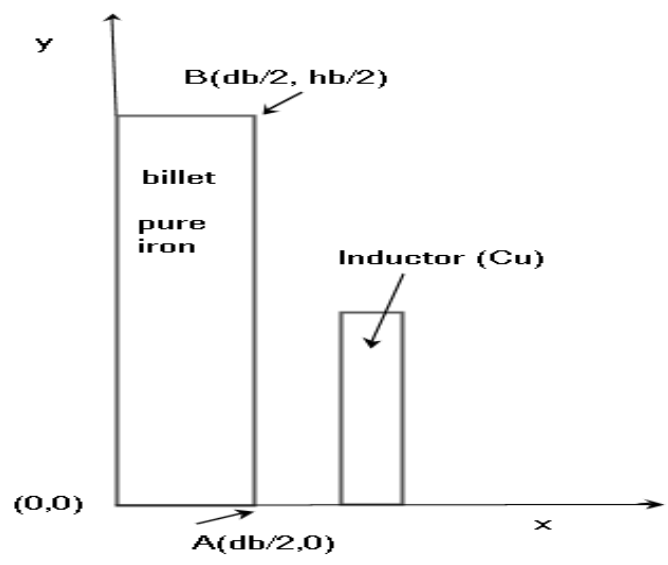

Figure 2 The quarter of studied system. 


\subsection{Equations}

The studied system presents an axisymmetry around the axis of load. Thus, only a quarter is used for calculus. The electrical conductivity of the load depends on temperature . The temperatures of study in Kelvin are : 298 ( $\left(25^{\circ} \mathrm{C}\right), 398$, 498 , 598 , 698 , 798 , 898 , 950 , 998 , 1008 , 1018 , $1028,1038,1040,10421042.95$. For each temperature, with a script Matlab, one determines the reduced magnetisation of saturation $m_{s}$, then the magnetization density $M$, then the magnetic susceptibility $\chi$, then the relative permeability $\mu_{r}$, then the relative reluctivity $v_{r}$. The Figure 3 represents $\mu_{r}$ as a function of temperature for temperatures in $\mathrm{K}: 298$ (top),398, 498, 598, 698, 798, 898, and 1042.95 (bottom).

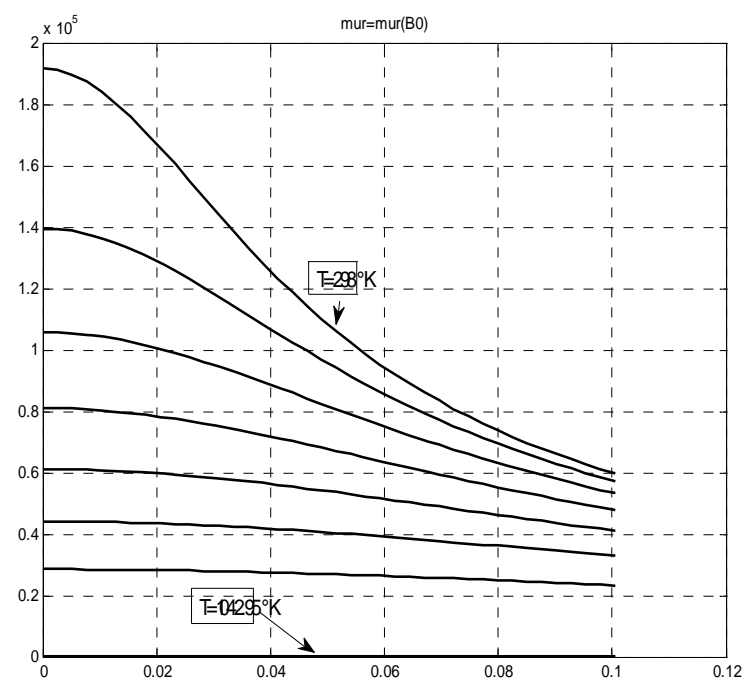

Figure 3. Relative permeability $\mathrm{m}_{\mathrm{r}}$ versus $\mathrm{B}_{0}$

For each temperature, $v_{r}$ instead $\mu_{r}$, was interpolated by a polynomial with $\mathrm{R}^{2}=1$. For example, for $\mathrm{T}=298 \mathrm{~K}$, the relative reluctivity is

$v_{r T 298}=p_{1} B_{0}^{4}+p_{2} B_{0}^{3}+p_{3} B_{0}^{2}+p_{4} B_{0}+p_{5}$

where $\mathrm{p}_{1}=0.04877, \mathrm{p}_{2}=-0.01696, \mathrm{p}_{3}=0.002388, \mathrm{p}_{4}=-3.256 \times 10^{-6}, \mathrm{p}_{5}=5.224 \times 10^{-6}$, and $B_{0}=\mu_{0} H_{0}$ is the applicated magnetic induction. Figure 4 represents $v_{r T 298}=\mathrm{f}\left(\mathrm{B}_{0}\right)$

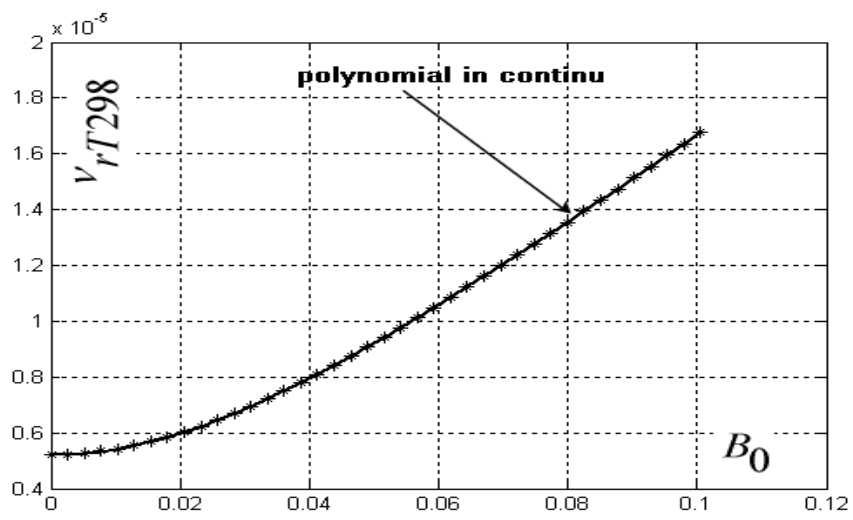

Figure 4. Relative reluctivity at $\mathrm{T}=298 \mathrm{~K}$ versus $\mathrm{B}_{0}$ 
The studied system presents an axisymmetric along Oy. The appropriate parameter in this case is the complex magnetic vector potential $\bar{A}$. Combining equations from (1) to (9), and taking in account the quasi-stationnarity of the phenomen, the system of partial differential equations governing our installation is [4]:

$\left\{\begin{array}{l}A_{-} r e: \operatorname{div}\left(v_{0} \cdot v_{r} \cdot \operatorname{grad}\left(A_{-} r e\right)\right)+\omega \cdot \gamma \cdot A_{-} i m+n \cdot J s_{-} r e=0 \\ A_{-} i m: \operatorname{div}\left(v_{0} \cdot v_{r} \cdot \operatorname{grad}\left(A_{-} i m\right)\right)+\omega \cdot \gamma \cdot A_{-} r e+n \cdot J_{-} \_i m=0\end{array}\right.$

where $A_{-} r e$ and $A_{-}$im are respectively real and imaginary components of the complex magnetic vector potential $\bar{A} \cdot v_{0}=$ vacuum reluctivity, the inverse of vacuum permeability. $v_{r}=$ relative reluctivity of billet interpolated for each temperature like in formula (13). $\omega=2 \pi f$ is the pulsation of the current source in the inductor. $J s_{-} r e$ and $J s_{-} i m$ are respectively real and imaginary components of the complex source current density. $n=$ a Boolean variable to identify regions carrying current. Through the boundary conditions, $n=1$ in the inductor and $n=0$ in the billet. The real and imaginary components of the complex current source density $\bar{J}$ s are calculated as [4]:

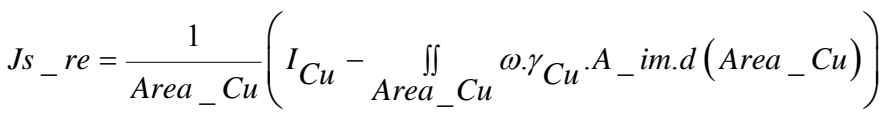

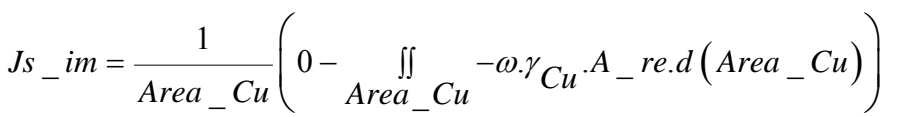

where $I_{C u}=6600 \mathrm{Amp}$ is the courant in the inductor, Area ${ }_{-} \mathrm{Cu}$ and $\gamma_{\mathrm{Cu}}$ are its area and electric conductivity .

The real and imaginary components of the complex current density in the iron or billet $\bar{J}_{b}$ are calculated as [4]:

$$
\begin{aligned}
& J_{b_{-} r e}=\frac{1}{\text { Area_Iron }}\left(0-\iiint_{\text {Area_Iron }}^{\int \cdot \gamma_{\text {Iron }} \cdot A_{-} i m \cdot d(\text { Area_Iron })}\right) \\
& J_{b_{-} i m}=\frac{1}{\text { Area_Iron }}\left(0-\underset{\text { Area_Iron }}{\iint}-\omega \cdot \gamma_{\text {Iron }} \cdot A_{-} \text {re.d } d(\text { Area_Iron })\right)
\end{aligned}
$$

where Area_Iron and $\gamma_{\text {Iron }}$ are area and electric conductivity of the billet. This electric conductivity as a function of temperature is

$$
\gamma_{\text {Iron }}=\frac{95.24 \times 10^{6}}{1+0.0045 T}
$$

The volumic power, in $\mathrm{W} / \mathrm{m}^{3}$, is calculated as

$$
P_{v}=\frac{\left|\bar{J}_{b}\right|^{2}}{\gamma_{\text {Iron }}}
$$

\section{Results and simulations:}

All equations from (14) to (20) were implemented in a script running under FlexPDE which is a code of computation using Finite Element Method and supporting large non-linearities in partial derivatives differential equations. The studied system presents a revolution's symmetry, thus, the calculus were made in $2 \mathrm{D}$ only.

The use of the relative reluctivity $v_{r}$ was preferred to the use of the relative permeability $\mu_{r}$ because $\mu_{r}$ is in the denominator of equations (14) and its polynomial interpolation gives a 
polynomial at the denominator. On the other hand, the polynomial interpolation of $v_{r}$ gives a polynomial in the numerator of equations (14), and then, the time of computation is reduced: some dozens of minutes versus two or three hours with the same computer. The Figure 5 , for $\mathrm{T}=950 \mathrm{~K}$ and $v_{r}=v_{r T 950}$, summarize some interesting physical parameters concerning this study.

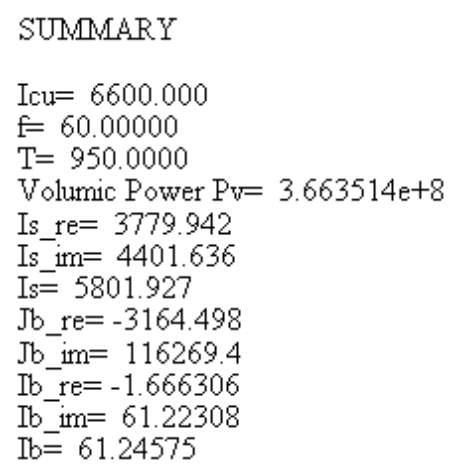

Figure 5. Some pertinent data calculated by the script FlexPDE.

$I_{b}$ is the magnitude of the current billet at $\mathrm{T}=950 \mathrm{~K}$ and with $v_{r}=v_{r T 950}$.

The problem has two lines of symmetry, hence a quarter of the problem is modelled. The appropriate unknown quantity is the magnetic potential vector, because it has only one component $A_{\varphi}$, in cylindrical coordinates, noted here $A$

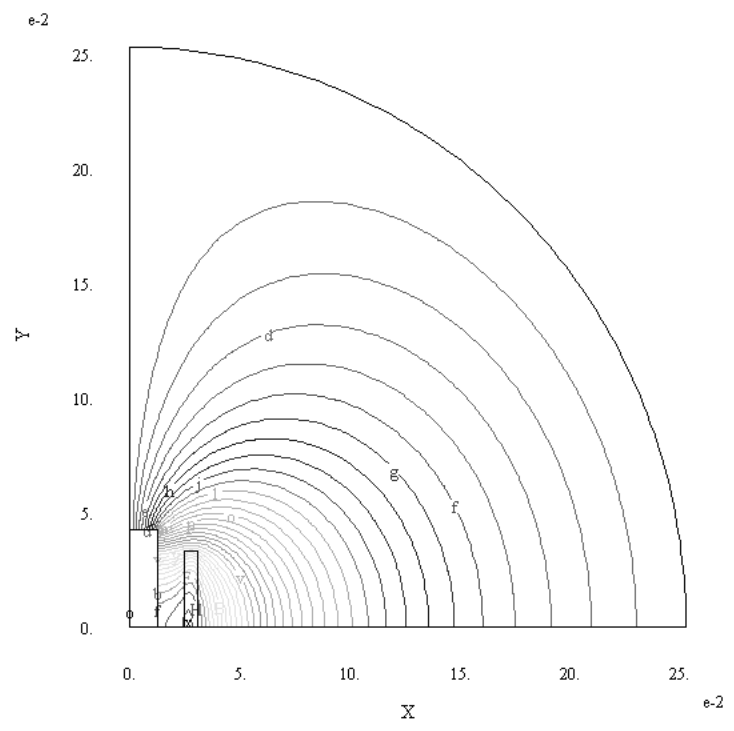

Figure 6. Field lines of the magnetic potyential vector at $\mathrm{T}=298 \mathrm{~K}$ and $. v_{r}=v_{r T 298}$

One of the interesting quantity in the induction heating is the volumic power in the load. Figure 7 represents this quantity from point A to point B like in Figure 2. 


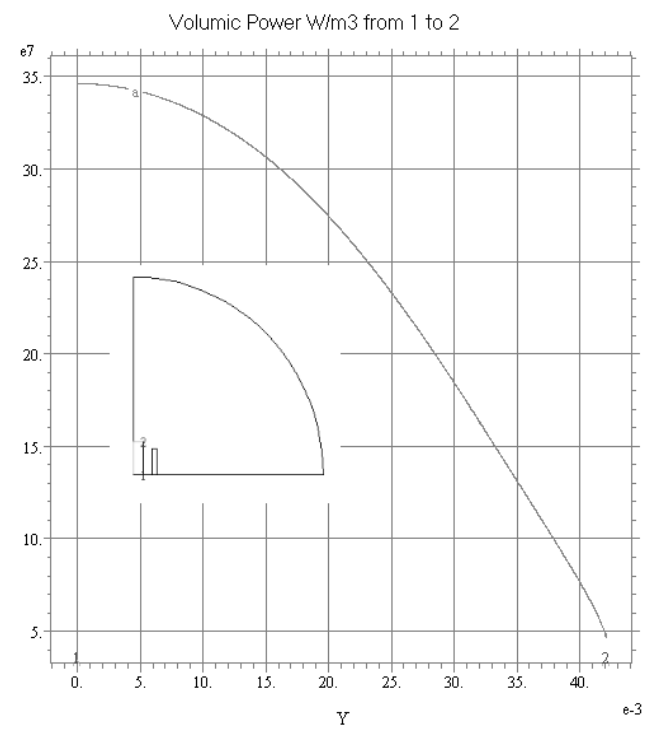

Figure 7. Volumic power along the segment $\mathrm{AB}$, at $\mathrm{T}=298 \mathrm{~K}$ and $v_{r}=v_{r T 298}$

The variation of the magnetic potential vector along the quarter of the billet is plotted in Figure 8 . It shows that the point 1 , or $\left.A\left(d_{b} / 2,0\right)\right)$, receives the maximum of the power.

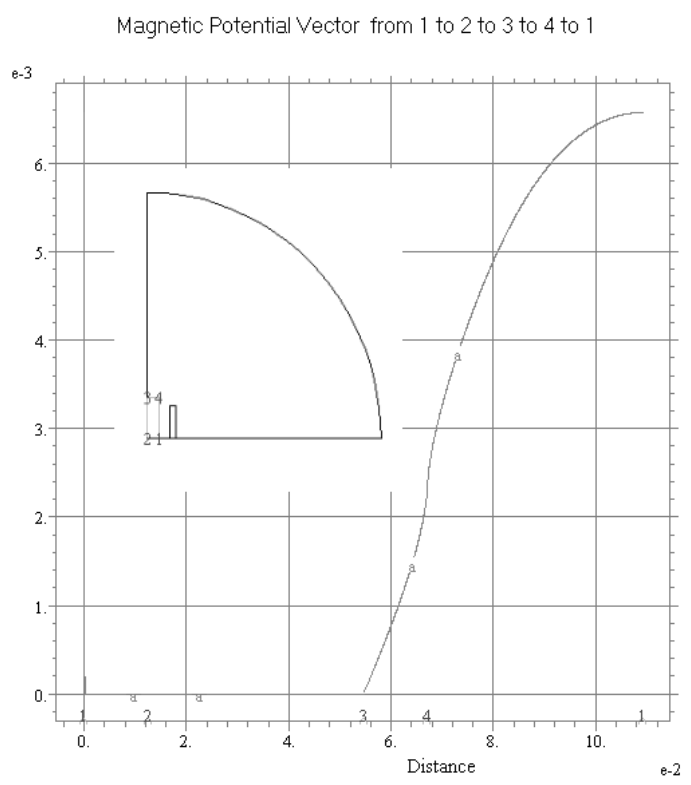

Figure 8. Magnetic potential vector along the perimeter 1 to 2 to 3 to 4 to 1

The study of the volumic power as a function of temperature is plotted in Figure 9, for the sixteen temperatures in ${ }^{\circ} \mathrm{K}: 298\left(25^{\circ} \mathrm{C}\right), 398,498,598,698,798,898,950,998,1008,1018$, $1028,1038,1040,10421042.95$. The same study takes in account the variation of the electric conductivity with the temperature in accordance with Formula (19). For each temperature, the relative reluctivity is interpolated by a polynomial like in Formula (13). Until about $1025 \mathrm{~K}$, the volumic power increases linearly with the temperature. But, near and before the temperature of Curie (1043K), the volumic power increases strongly. A special study in this zone is required. After the point of Curie, the pure iron becomes amagnetic, i.e. $v_{r}=1$. 


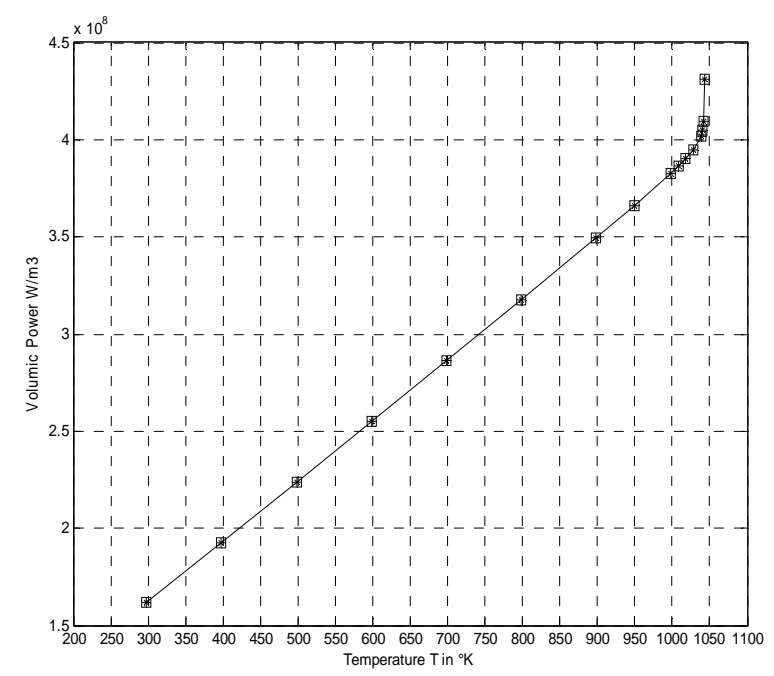

Figure 9. Variation of volumic power with temperature.

The electric current in the billet follows the same comportment that the volumic power. Figure 10 shows its variation with temperature. The script allows to have the real and imaginary currents in the inductor as well as in the load. Then, we can calculate their magnitudes and their phases. A study with varying the current frequency presents also an interest because it is known that the transmitted power towards the load increases when the frequency increases.

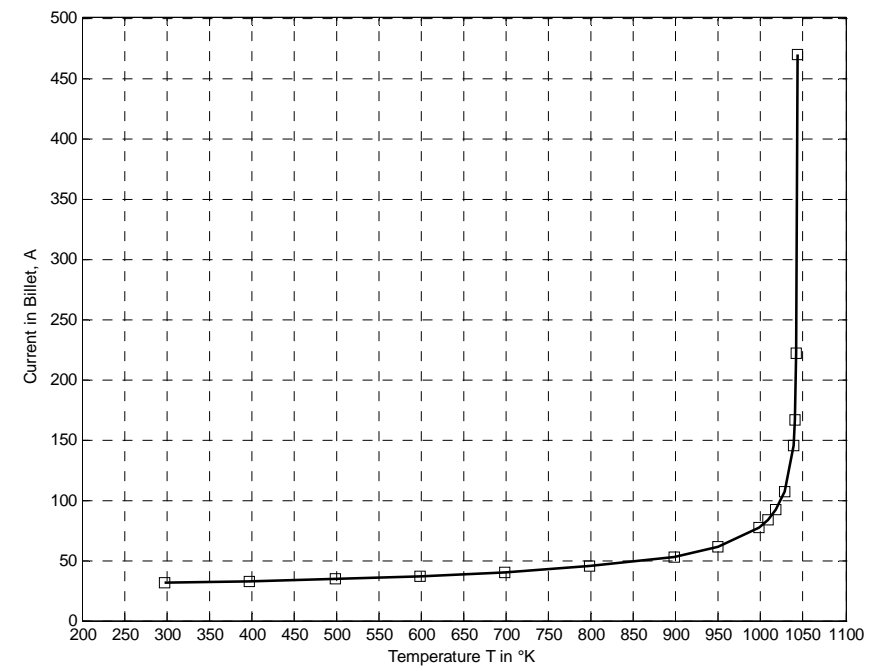

Figure 10. Variation of the electric current in the load with temperature.

\section{Conclusion:}

The conception and optimization of devices in Electroheat, require, first an thermic analysis and after, an electomagnetic one. The relative reluctivity was modelled as a function of the applicated magnetic field, but also a function of the temperature in the case of the pure iron. The variations of the volumic power and the electric current in a billet was established. Those variations show a large augmentation of the two quantities near the Curie's temperature. The results of simulations obtained here with the script FlexPDE are reliable because the same code was used in other applications with succes. 


\section{References:}

[1] Ch. Kittel : Introduction à la Physique de l'État Solide. Dunod. 1972. Paris.

[2] B. Merouane, M. Sebbani : Modelisation of permeability as a function of induction and temperature by the Brillouin's function for the formalization of the magnetothermal problem. Advancved Materials Research Vols.941-944 (2014) pp 677-683 (C) (2014) Trans Tech Publications. Switzerland.doi: 10.4028/www.scientific.net/AMR.941-944.677

[3] B.Merouane, M. Sebbani : Modelisation of calorific capacity by the Debye and Brillouin functions for the formalization of the magnetothermal problem. Advancved Materials Research Vols.941-944 (2014) pp 2449-2456 (C) (2014) Trans Tech Publications. Switzerland. doi: 10.4028/www.scientific.net/AMR.941-944.2449

[4] B.Merouane : Distribution des champ électromagnétique et thermique dans une billette placée dans un four à induction. Thesis, Electroheat Laboratory, University of Sciences and Technology of Oran, Algeria. 